\title{
The building of BODHI, a bio-diversity database system
}

\author{
Srikanta J. Bedathur*, Jayant R. Haritsa, Uday S. Sen \\ SERC, Database Systems Lab, Indian Institute of Science, Bangalore 560012, India
}

\begin{abstract}
We have built a database system called BODHI, intended to store plant bio-diversity information. It is based on an object-oriented modeling approach and is developed completely around public-domain software. The unique feature of BODHI is that it seamlessly integrates diverse types of data, including taxonomic characteristics, spatial distributions, and genetic sequences, thereby spanning the entire range from molecular to organism-level information. A variety of sophisticated indexing strategies are incorporated to efficiently access the various types of data, and a rule-based query processor is employed for optimizing query execution. In this paper, we report on our experiences in building BODHI and on its performance characteristics for a representative set of queries.
\end{abstract}

\section{Introduction}

Over the last decade, there has been a revolutionary change in the way biology has come to be studied. Computer assisted experimentation and data management have become commonplace in the biological sciences and the branch of BioInformatics is drawing the attention of more and more researchers from a variety of disciplines. A key area of interest here is the study of the biodiversity of our planet. The database research community has also realized the exciting opportunities for novel data management techniques in this domain - in fact, bio-diversity was featured as the theme topic at the Very Large DataBase (VLDB) 2000 Conference [1].

Over the last 3 years, we have built a database system, called BODHI (Bio-diversity Object Database arcHItecture $)^{1}$, that is specifically designed to cater to the special needs of biodiversity

\footnotetext{
*Corresponding author.

${ }^{1}$ Gautama Buddha gained enlightenment under the Bodhi tree.
}

applications. While BODHI currently hosts purely plant-related data, it can be easily extended to supporting animal-related information as well. In this paper, we report on our experiences in building BODHI, and also present its performance profile with regard to a representative set of user queries.

\subsection{Background}

The study of bio-diversity, as outlined by the World Conservation Monitoring Center (WCMC) [2], is an integrated study of Species, Ecosystem and Genetic diversity. The data associated with these domains vary greatly in the scale of their structural complexity, their query processing cost, and also their storage volume. For example, while the taxonomy information of species diversity has complex hierarchical structure, spatial data and spatial operators associated with ecosystem diversity are inherently voluminous and computationally expensive. On the other hand, genetic diversity 
is based on specialized pattern recognition and similarity identification algorithms over DNA or protein sequences of the species. Thus, supporting such diverse domains under a single integrated platform is a challenge to the data management tools currently used by the ecologists. More often than not, these scientists make use of different tools for managing and querying over each of the domains, leading to difficulties in performing cross-domain queries.

To illustrate the above point, consider the following target query, which is of interest to modern evolutionary biologists and similar to those that have appeared in the ecological literature (for example [3]):

Query 1. Retrieve names of all fruit-bearing shrubs that share a part of their habitats and have a chromosomal DNA sequence score of over 70 with Magnolia champa.

The above query is typical in the new age of biodiversity studies, where researchers are simultaneously studying the macro-level and micro-level relationships between various target species. Answering the query requires the ability to perform integrated queries over taxonomy hierarchies ("fruit-bearing shrubs"), recorded spatial distribution of species ("common habitat"), and the genome sequence databases ("Chromosomal DNA sequence score above 70"). Unfortunately, however, due to the lack of holistic database systems, biologists are usually forced to split the query into component queries, each of which can be processed separately over independent databases, and then either manually or through a customized tool perform the join of the results obtained from the component queries.

For example, a typical "experience story" for answering the above query, as gathered from domain experts, would be:

(1) Locate all fruit-bearing shrubs by performing a selection query over the taxonomy database, stored in MS-Access [4], a ubiquitous PCbased relational database, and retrieve the keys for their habitats.

(2) For all the keys output in Step 1, access the associated habitat data, stored as polygons in ArcView [5], a popular spatial database product. Then, for each qualifying polygon, find all the habitats in the spatial database that intersect this polygon. Finally, compute an intersection between the original set of polygons and the newly derived set of polygons in order to prune away the habitats of organisms other than fruit-bearing shrubs.

(3) From the output of Step 2, identify the names of the species of the target shrubs, and then perform repeated BLAST [6] searches over the EMBL GenBank [7] DNA sequence database to identify the sequences (and, thereby the species), that have a score of more than 70 . Note that this final score-based pruning has to be performed externally by the researcher.

Long procedures, such as the above, for answering standard queries are not only cumbersome but can also lead to delays in understanding various microlevel and macro-level bio-diversity patterns. Even worse, the patterns may not be found at all due to limited human capabilities (an example of this problem was reported in the molecular biology study of [8], where comparison of sequences "by hand" missed out some of the significant alignments thereby leading to erroneous conclusions about the functional similarity of the proteins examined in the study).

\subsection{The BODHI system}

Based on the above discussion, there appears to be a clear need for building an integrated database system that can be productively used by the biodiversity community. To address this need, we have built the BODHI database system in association with the ecologists and biologists at our institute. The project has been funded by the Department of Biotechnology, Ministry of Science and Technology, Government of India.

BODHI is a native object-oriented system that naturally models the complex objects ranging from hierarchies to geometries to sequences that are intrinsic to the bio-diversity domain. In particular, it seamlessly integrates taxonomic characteristics, spatial distributions, and genomic sequences, 
thereby spanning the range from molecular to organism-level information. To the best of our knowledge, BODHI is the first system to provide such an integrated view.

BODHI is fully built around publicly available database components and system software, and is therefore completely free. In particular, the SHORE micro-kernel [9] from the University of Wisconsin (Madison) forms the back-end of our software, while the $\lambda$-DB extensible rule-based query optimizer [10] from the University of Texas (Arlington) is utilized for production of efficient execution plans. The system is currently operational on a Pentium-III-based PC hosting the Linux operating system.

A variety of sophisticated access structures, drawing on the recent research literature, have been implemented to provide efficient access to the various data types. For example, the Path-Dictionary [11] and Multi-key Type indexes [12] accelerate access to inheritance and aggregation hierarchies, while the $\mathrm{R}^{*}$-tree [13] and Hilbert $\mathrm{R}$ tree [14] are used for negotiating spatial queries.

The BODHI server is compliant with the ODMG standard [15], supporting an OQL/ODL query and data modeling interface. To enable biologists to interface with the system in a more intuitive manner, BODHI also supports access through the Web client-server model wherein clients submit requests through the HTTP protocol and CGI-bin scripts, and the results are provided through the browser interface. Further, the server is "XML-friendly", outputting the result objects in XML format, enabling clients to visualize the results in their favorite metaphor.

We view BODHI's role as not merely that of a database system in isolation, but as a central repository that provides a common information exchange platform for all the tools used in a biologist's "data workbench" such as decision support systems, visualization packages, etc. That is, BODHI occupies a role similar to that played by the Management Information Base (MIB) in tele-communication network management.

Algorithms proposed in the research literature typically tend to be evaluated in isolation and it is never clear whether their claimed benefits really carry through in practice with regard to end-user metrics in complete systems. We suggest that researchers may find it possible to address this deficiency by using BODHI as a "test-bed" on which new ideas can be evaluated in a real-world kind of setting. As reported later in this paper, we have ourselves carried out this exercise with regard to spatial indexes.

Finally, BODHI is living proof that developing a viable biological DBMS does not necessarily entail expensive hardware or software but can be cobbled together using commodity components.

In this paper, we report on our experiences in building BODHI, and also present its performance profile with regard to a representative set of biological queries (including Query 1 mentioned above). ${ }^{2}$ Since, as mentioned earlier, there are no comparable systems that we are aware of, for the most part our results can be placed only in an absolute perspective. However, specifically for queries restricted solely to spatial data, we were able to utilize the well-known Sequoia 2000 benchmark [17], and additional spatial aggregate operators such as Closest introduced in the [18]. Here, our numbers are competitive with those obtained by the Paradise GIS system [18], that was highly optimized for handling only spatial queries.

\subsection{Contributions}

To summarize, the main contributions of this paper are the following:

First, we present the architecture and implementation of a high-performing object database system tuned specially for the needs of the biodiversity research community. To the best of our knowledge, this is the first such system supporting diverse data domains ranging from genomic sequences to geographical features, and allowing queries that span across these domains.

Second, we show that BODHI is comparable in performance to other special purpose data management systems by evaluating its spatial data handling, involving computationally expensive operations, against Paradise, a high performance spatial data management system.

\footnotetext{
${ }^{2}$ A preliminary position paper focusing solely on the BODHI architectural design was presented in [16].
} 
Finally, through a detailed performance study we show that genomic sequencing queries are extremely expensive to compute, even more so than spatial operations, highlighting the urgent need for developing efficient sequence indexing strategies.

\subsection{Organization}

The remainder of the paper is organized as follows: Desirable design goals for bio-diversity DBMS are laid out in Section 2. The BODHI system architecture and its implementation are covered in Sections 3 and 4, respectively. Then in Section 5, we present our experiences in building BODHI, and followup with a detailed performance evaluation in Section 6. Related work is reviewed in Section 7. Finally, in Section 8, we present our conclusions and future research avenues.

\section{Design goals}

In this section, we highlight the main features that would be desirable in a bio-diversity information system. These include efficient handling of complex data types, facilities for molecular pattern discovery, and user-friendly interfaces, described in more detail below.

\subsection{Handling of complex data types}

Plant bio-diversity data can be broadly classified into the following three categories:

Taxonomy data: This is data about the relationships between species based on their characteristics. This includes phenetic relationships (based on comparison of physical characteristics) and phylogenetic relationships (based on evolutionary theory) [19]. The various characteristics on which these relationships depend may vary in time due to discovery of a new class of characteristics, corrections to previously recorded characteristics, etc.

Geo-spatial data: The study of ecology of species involves recording the geographical and geological features of their habitats, water-bodies, artificial structures such as highways which might affect the ecology, etc. These are represented on a map of the region and have to be handled as spatial data by the database.

Bio-molecular data: The genetic makeup of species is becoming increasingly important with a large number of genome sequencing projects working on organisms and plants. For example, "bio-prospectors" look for indigenous sources of medicines, pesticides and other useful extracts. Such data can be discovered from the biomolecular and genetic composition of species.

The above data-types have complex and deeply nested relationships within and between themselves. Further, they may involve sophisticated structures such as sequences and sets.

\subsection{Molecular pattern discovery}

The molecules that are of interest in biodiversity are DNA and proteins. DNA is represented as a long sequence based on a four nucleotide alphabet. There are regions in the DNA sequence, called exons, which contain the genetic code for the synthesis of proteins. The proteins are long chains of 20 amino acids. Each protein is characterized by the amino acid patterns it has, and is responsible for various functionalities in a cell which determine the characteristics of the organism or plant.

The similarity between two genetic sequences is a measure of their functional similarity. Analysis of DNA and protein sequences from different sources gives important clues about the structure and function of proteins, evolutionary relationships between organisms, and helps in discovering drug targets.

There are a number of popular algorithms, such as Dynamic Programming, BLAST [6], FastA [20] etc., for performing the similarity search over genetic sequences. Researchers and bio-prospectors frequently search the database using these algorithms to locate gene sequences of interest. However, the implementation of these algorithms is typically external to the database, making them relatively slow. It therefore appears attractive to consider the possibility of integrating these algorithms in the database engine (this observation is gaining currency in the commercial database arena 
as well, as exemplified by IBMs provision of homology searching through UDFs in DB2 [21]).

\subsection{Usage interface}

As with all other scientific communities, the biodiversity community relies on timely knowledge dissemination. Therefore, supporting access through the Internet is vital for maximizing the utility of the information stored in the database.

Typically, bio-diversity data is autonomously collected and managed by individual research institutions and commercial enterprises. In order to improve data availability, it is necessary that such localized and autonomous data repositories be able to exchange data. The current state of information exchange amongst various bio-diversity data repositories is not very satisfactory [22]. However, with the advent of XML, many research groups are proposing DTDs in individual fields of ecology and genetics [23,24]. The bio-diversity information system should support these DTDs for handling data over heterogenous set of repositories.

It is imperative to have a good visualization interface for the results produced by the system since (a) the end-users are biologists, not computer scientists and (b) the results could range from simple text to multi-dimensional spatial objects.

Finally, most of the research in bio-diversity is done by small teams of researchers who work within low budgets and are unable to afford highcost data repository systems. Therefore, solutions that are completely or largely based on publicdomain freeware which can be hosted on commodity hardware, with total cost not exceeding $\$ 1000$, are essential for these groups.

\section{Architectural overview of BODHI}

As mentioned earlier, bio-diversity data is inherently hierarchical and has complex relationships. In order to enable natural modeling of these entities and their relationships, BODHI is designed as an object oriented database server, with OQL/ ODL query and data modeling interfaces. While we consciously adopted this technology from the very beginning of our project in 1998, it is gratifying to note that the same approach is now being taken by large-scale biological repositories such as European Molecular Biology Laboratory (EMBL) - in a recent report, they have indicated their intention in moving from their current Oracle-based relational database system to an object-based data management and distribution scheme for their massive genomic databases [25].

The overall architecture of BODHI is shown in Fig. 1. At the base is the storage manager, which provides the fundamental needs of a database server such as device and storage management, transaction processing, logging and recovery management. The application-specific modules, which supply the taxonomic, spatial and genomic services, are built over this storage manager and form the functional core of the system. The query processor interfaces with the functional modules and performs query processing and optimization using statistics exported by these modules. Finally, the client interface framework receives query forms over the Internet from clients and returns results in the desired format. In the remainder of this section, we describe the core database components in more detail.

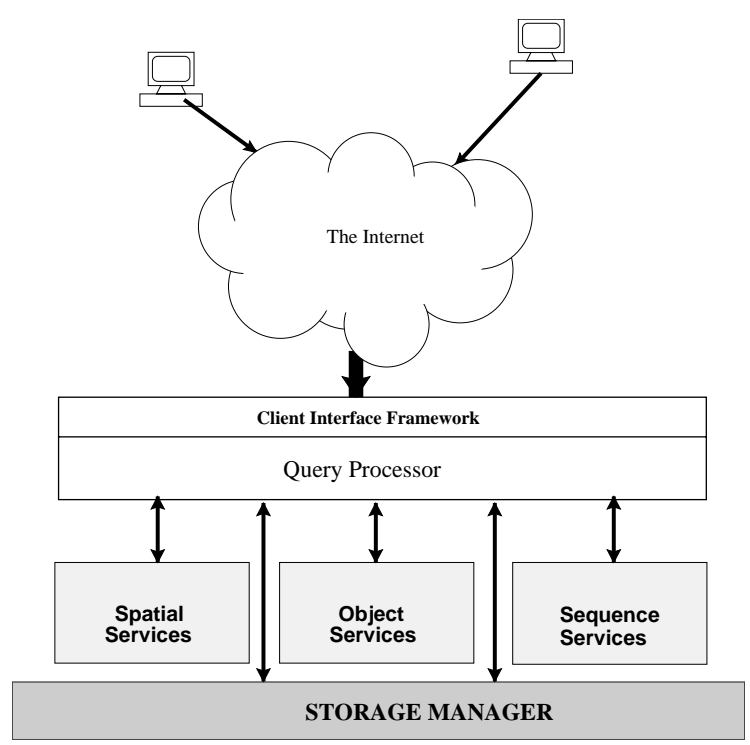

Fig. 1. Schematic of architecture of BODHI. 


\subsection{Service modules}

The three service modules: Object Services, Spatial Services and Sequence Services, provide the functionalities for each of the bio-diversity data domains mentioned in the Introduction, and are described in more detail below.

Object Services: While the storage manager handles basic object management, it is necessary to support specialized access methods for efficient processing of queries over the object schema and its instantiation. The Object Services component bundles together these access methods.

In querying over object oriented data models, it is common for predicates to follow arbitrarily long (sometimes recursive) relationship paths, or be evaluated over an inheritance hierarchy rooted at a chosen base type. As illustrations, consider the following query types over a typical bio-diversity data model such as that given in Fig. 2, which captures the taxonomic, spatial and genomic components:

(1) Identify the PlantSpecies based on one or more of its IdentCharacteristics.
(2) Retrieve all IdentCharacteristics of a given PlantSpecies.

(3) List the names of all PlantSpecies associated with a GeoRegion.

The above queries illustrate the fact that queries over relationship graphs of bio-diversity data models may have either an ancestor class or a nested class as the predicate, and might need to be evaluated over an inheritance hierarchy. These queries may involve joins between extents of objects in the traversal paths, or scanning over multiple extents for the predicate in the case of queries over type hierarchies. Therefore, access methods for both inheritance and aggregation hierarchies are included in this module.

Spatial Services: Spatial (or geographic) data, in both vector (object) and raster (bitmap) formats, constitutes the bulk of the bio-diversity information. Due to the inherent complexity of spatial operations (such as overlap, closest, etc.), combined with large volumes of data, spatial query processing is considered to be a major bottleneck in the expeditious processing of a cross-domain query (such as Query 1 in Section 1).

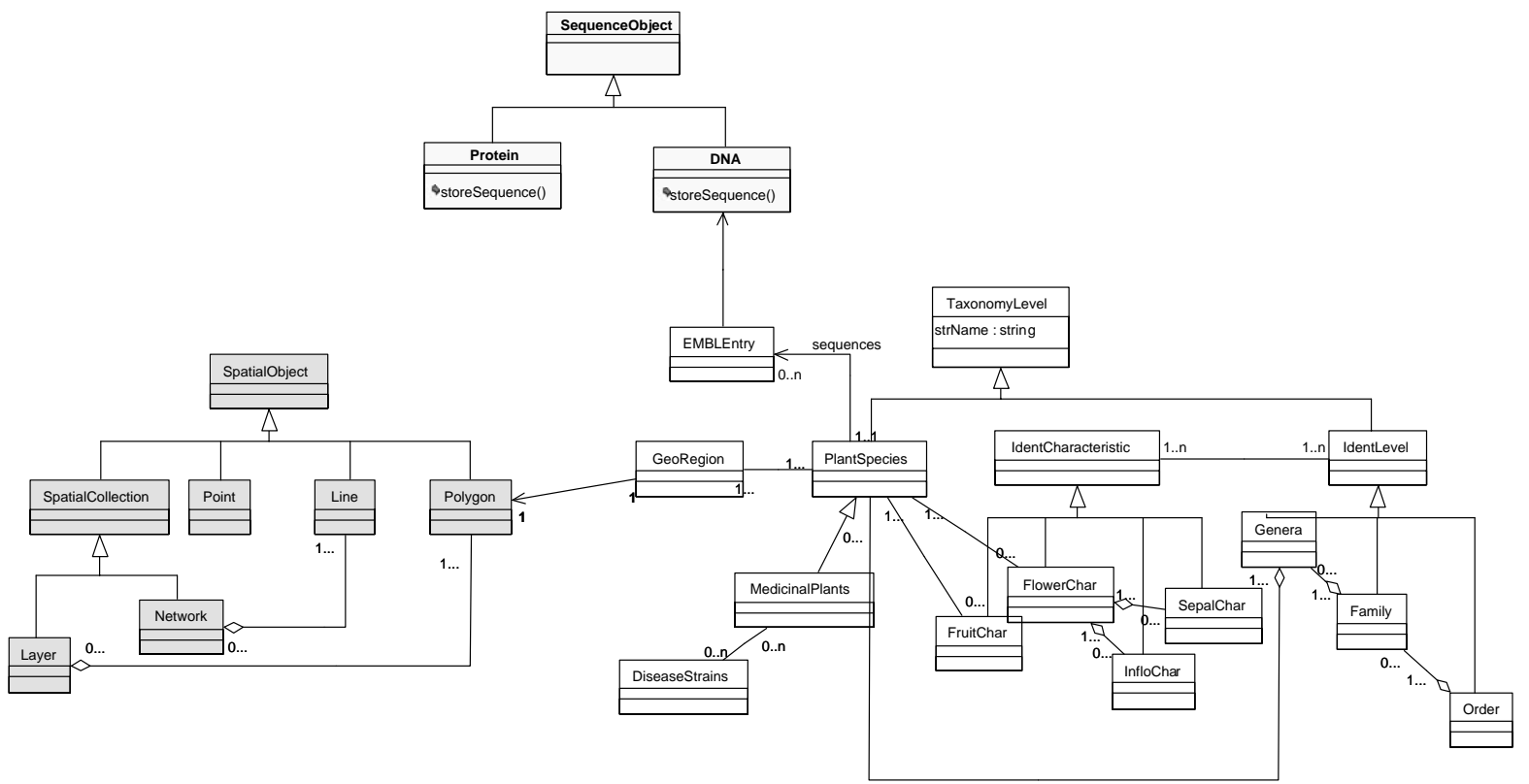

Fig. 2. Bio-diversity object model. 
The Spatial Services module provides efficient implementations of access methods and spatial operations.

To ensure that the access methods have efficient disk allocations, and thereby alleviate the performance bottleneck mentioned above, these methods are built within the storage manager. While this choice makes it cumbersome to replace or upgrade the storage manager, we felt that the resulting performance benefits would outweigh the disadvantages.

The Spatial module provides a spatial-type system based on the ROSE Algebra [26]. These types, whose hierarchy is shown in Fig. 2, consist of Simple primitives: Point, Polyline, and Polygon; and Compound primitives: Layer and Network, which are collections of related Polygons and Polylines, respectively.

Sequence Services: In modern bio-diversity studies, genetic data plays an important role [3]. The Sequence Services module interfaces with the storage manager to provide efficient storage of genetic sequences and sequence retrieval algorithms such as BLAST, FasTa, etc. These algorithms are expensive to compute since there are currently no obvious ways of caching or indexing to speed up their computation, and a full scan of the sequence database is therefore entailed each time. The Sequence Services module uses appropriate storage structures for efficient execution of the genetic algorithms.

This module supports two primitive types: $D N A$ and protein. The DNA alphabet of 4 nucleotides is encoded using 2 bits and similarly the protein sequence alphabet of 20 is encoded in 5 bits. The functions for translation of DNA sequences into and from protein sequences for complementary DNA strand generation, and for substring operations are also included in this module. Finally, the alignment-based sequence similarity algorithms such as BLAST (using standard scoring matrices like BLAST or BLOSUM) are also part of the module.

\subsection{Query System}

The data modeling and query language for BODHI is based on the ODL and OQL languages, respectively, from the ODMG standard [15]. These languages have been enhanced with support for both the typesystems over spatial and genetic data, and the operators over these typesystems.

The query processor contains, in addition to the techniques available in generic database systems, specialized optimization schemes for:

- Spatial operators, when spatial indexes are available on predicate attributes.

- Relationship path traversals.

- Queries over a type hierarchy of the data model.

The presence of user defined methods in the synthesized object types (for example, Print method on objects, Area over polygons, etc.), form a serious obstacle for optimal plan generation, since their costs are not directly available to the query optimizer. A variety of strategies for handling this situation have been proposed in the literature [27,28]. In BODHI, we have extended the ODL language to allow optional definition of cost functions, and functionally equivalent methods. These extensions enable the cost-based optimizer to compute the cost associated with each of the equivalent methods, before choosing the best execution strategy.

Client Interface Framework: The client interfacing is an important layer in the query interface of BODHI. We have developed a simple framework to transform the objects of the query results into formats amenable for transportation to endclients. With clients following different needs for their visualization and query capabilities, we feel this becomes an important part of the query interface. Using this framework, users can easily implement their transformation rules which are then applied to the appropriate objects in the query results. The transformed results are then shipped to the clients.

\section{Implementation choices}

In this section, we highlight the important software choices that we had to consider in BODHI, and provide the rationale for the decisions that we made. We discuss these choices under the following heads: (i) selection of storage 
manager and query processor, (ii) selection of access methods, and (iii) positioning of implementation components.

\subsection{Selection of storage manager and query processor}

For the back-end storage manager, we selected the SHORE system [9] developed at the University of Wisconsin (Madison) which, at the time we began the project in late 1998, had a major release the previous year that was operational on both Solaris and Linux platforms. We were drawn towards SHORE due to its attractive array of features, including:

- Well-implemented support for basic database functionalities such as transactions, logging and recovery management, device and storage management, etc. Recovery is implemented through the ARIES algorithm [29] which has become the de-facto industry standard, while multi-granularity locking is provided for enhanced concurrency.

- Integrated file-system interface with DBMS functionality. This can be extremely useful in handling genomics data which is available largely as flat-files.

- First-class support for user defined types.

- Availability of a framework for writing Value Added Servers (VAS) - to provide additional features to the storage manager.

- Presence of $R^{*}$-Tree [13], a spatial indexing structure built within the SHORE kernel (in addition to the standard $B^{+}$-Tree index).

- Availability of source code, which enabled us to enhance many of the features of SHORE (the version we have used is Version 1.1.1, which was the latest at the time we began our project).

- Successfully tested under at least two large scale research prototypes [18,30].

- Intrinsic support for parallelism on a multiprocessor or network of workstations.

After we had been into development for about a year, we had reached the stage wherein we were thinking about the implementation of the query processor. In particular, we were considering the possibility of building our own query processor, using either a Volcano-style framework or a Tigukat-style framework. We dropped this idea, however, when news broke (on the dbworld [31] mailing list) of the first release of $\lambda$-DB [10], an extensible rule-based optimizer from the University of Texas (Arlington), which, serendipitously enough, had been implemented on Shore! This vastly reduced our design time on the query processor front. Further, $\lambda$-DB came with an attractive set of features including query transformation and optimization rules for OQL (specified using the OPTL optimization specification language), and a functional design that made it easy to enhance and specify additional rules. Finally, it had a firm mathematical foundation in monoid comprehension calculus that permitted optimizations similar to those found in relational query rewriting engines.

\subsection{Selection of access methods}

As discussed earlier, BODHI includes indexes for inheritance hierarchies, aggregation hierarchies, and spatial data that are implemented in the Object and Spatial Services modules. For each of these indexing categories, there have been numerous proposals in the research literature, requiring us to make a carefully selected choice.

We had intended to add indexes for sequence data as well. Unfortunately, however, until this issue was addressed very recently in [32,33], no practical solutions for indexing the sequences were available, rendering it impossible to realize our objective. We are now investigating the incorporation of these new methods in the BODHI system.

Inheritance hierarchies: For indexing inheritance hierarchies, we have chosen the Multi-key Type Indexing [12]: The basic idea behind MT-index is a mapping algorithm that maps type hierarchies to linearly ordered attribute domains in such a way that each sub-hierarchy is represented by an interval of this domain. Using this algorithm, MT-index incorporates the type hierarchy structure into a standard multi-attribute search structure, with the hierarchy mapped onto one of the attribute domains (type domain). This scheme supports queries over a single extent as well as over 
extents of classes under a subtree. This can also be extended to support the multi-attribute queries.

Apart from its simple transformation of the tree into a linear path, a major attraction of the MTindex is that it can be implemented using any of the multi-dimensional indexing schemes. In particular, since SHORE natively supports $R^{*}$-trees, the MT-index could be directly implemented using this structure, resulting in considerably reduced programming and integration effort.

Containment hierarchies: For indexing aggregation hierarchies, we have chosen the Path-Dictionary $(P D)$ index [11]. The PD-Index consists of three parts: the Path-Dictionary which supports the efficient traversal of the path, and the identity index and the attribute index which support associative search. The identity index and attribute index are built on top of the Path-Dictionary.

Conceptually, the Path-Dictionary extracts the compound objects, without the primitive attributes, to represent the connections between these objects in the aggregation graph. Since attribute values are not stored in the Path-Dictionary, it is much faster to traverse the nodes in the extracted Path-Dictionary. In order to support associative search based on attribute values, PD-Index provides attribute indexes which are built for each attribute on which there are frequent queries. When the identifier of an object is given, the path information is obtained using the identity index built over the PD.

On the positive side, the PD-index supports both forward and backward traversals of the hierarchy with equal ease; further, its performance evaluation indicated significantly improved access times in [11]. A limitation, however, is that it only handles 1:1 and 1:N relationships. Since typical schemas of bio-diversity databases include aggregations of N:M cardinality, and structures such as sets, bags and sequences in the aggregation path, we had to extend the implementation of the PDindex to handle these constructs as well.

Access methods for spatial data: For spatial data, SHORE natively supports the $R^{*}$-Tree [13], which is the most popular spatial access method since it achieves better packing of nodes and requires fewer disk accesses than most of the alternatives. However, a problem with the $R^{*}$-Tree is that even though it has tight packing to begin with, its structure may subsequently degrade in the presence of dynamic data. To tackle this, we implemented the Hilbert R-Tree [14], which is designed for handling the dynamic spatial data while maintaining good packing of the index structure. It makes use of a Hilbert space-filling curve over the data-space to linearize (i.e. obtain a total ordering of) the objects in the multi-dimensional domain space. A performance evaluation in [14] shows this structure to provide better packing in the presence of dynamic spatial data and thus better performance. However, the evaluation was considered in isolation and therefore one of the goals of our study was to investigate how well these performance improvements carried over to a real system.

\subsection{Positioning of implementation components}

In addition to selection of software and the indexing methods, another important decision that determines the system performance and extensibility is the placement of functionality in the implementation. One option is to achieve performance improvements by supporting every feature of the system at the lowest level-for example, by implementing at the SHORE storage manager level. However, this becomes a huge effort to extend and improve the system by addition of new basic types, new access structures, etc. At the same time, if we provide all the additional features at layers external to the storage manager then the overall performance could suffer. Therefore, we considered these two competing requirements of the system carefully while placing the implementation of the services, and aimed to optimize extensibility while minimizing the performance overhead on the system. (Fig. 3)

Object Services: As mentioned previously, this module bundles the Path-Dictionary and Multikey Type indexes over object aggregation and type hierarchies, respectively. The Path-Dictionary structure is implemented as a VAS, which maintains the path-dictionary on a data repositorywith its own recovery and logging facilitiesindependent from the main database. This gives the query processor an opportunity to scan the 


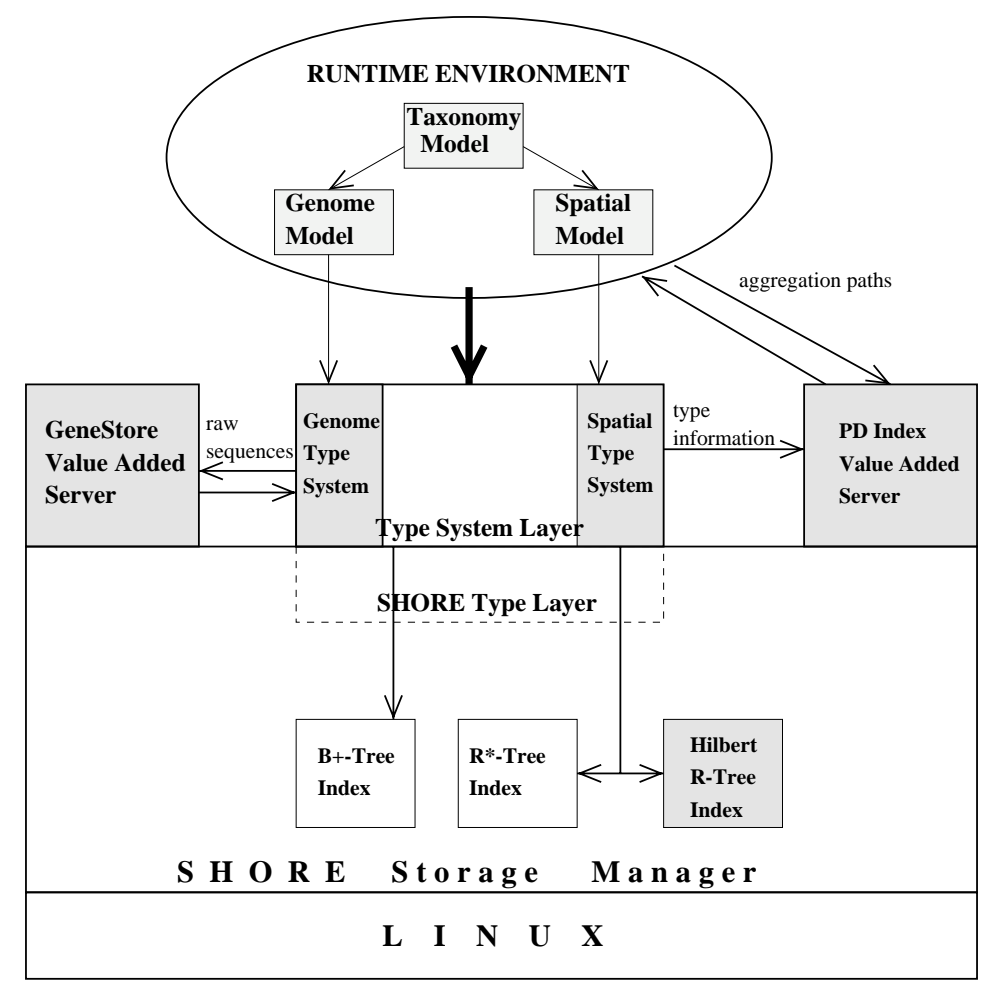

Fig. 3. Positions of implementation components.

path-dictionary repository in parallel to the other data scans active at the same time. Further, the locking overheads are distributed over different storage management threads.

The Multi-key Type index, on the other hand, is instantiated as an $R^{*}$-Tree, which is available for spatial indexing, with linearized-type system as a dimension and each object treated as a "point" in the spatial sense.

Spatial Services: In addition to the $R^{*}$-Tree provided by the Shore storage manager, the spatial services module provides the Hilbert R-Tree which is intended for use with highly dynamic spatial workloads. This index could be implemented as a VAS external to the database, utilizing the Shore SM interface which allows introducing new logical index structures. With this approach, however, no page-level storage control is provided, thereby making it infeasible to implement index structures such as Hilbert R-Tree that rely on physical packing of data for performance benefits. We were thus forced to implement the Hilbert R-Tree by refactoring the existing $R^{*}$-Tree implementation.

We had the option of implementing the spatialtype system, illustrated in Fig. 2, either as part of the basic-type system (similar to the support of types like integers, strings, references, etc.) or at the same level as a user defined-type system. In the former approach, we do gain the storage efficiency and low object creation overhead, but we lack the extensibility and ease of implementation available in the latter approach. The final choice was to go for an extensible-type system, that is, to provide the spatial-type system (along with sequence-type system - discussed below), as a user level library which can be modified and extended by the database administrator without having to work on the storage manager layers.

Sequence Services: The type system of the Sequence Services, consisting of DNA and Protein types, are provided in the same way as the spatial types, which we have described above. In addition, the DNA sequence type has extra requirements for 
its storage. The DNA sequences are usually very long (1000-10 000 basepairs), and consists of only four alphabets. Instead of storing them as character strings, we store them in a compressed form and perform queries over the compressed records rather than on the character strings. The efficient storage of the raw sequences is implemented as a separate VAS which provides advantages similar to those mentioned in the Path-Dictionary implementation.

\subsection{Implementation of user interface}

The user interface allows users to graphically construct OQL queries, and post them to the query processor through the web-server. These OQL queries are validated at the browser end, by javascripts associated with the query forms. The queries are received by the web-server through CGI extensions, which enable interaction between web-server and the BODHI query-processor.

The query-processor generates the output in XML [34], using semantic tags associated with each object in the result set. This representation can be visualized using a tool written on top of the browser, and enables users to visualize the results in their favorite metaphor.

A sample query input form and a sample tagged output are shown in the appendix.

\section{Experiences}

In the previous sections, we have described the architectural design of BODHI and the specific choices that we made for the various components of the design. We move on now to discussing the experiences and lessons that we learned during the course of implementing these choices in our prototype system. Some of the issues that we raise here with regard to SHORE and $\lambda$-DB have been addressed in subsequent releases of these codebases - we are constrained, however, to continue to use version 1.1.1 of Shore and version 0.3 of $\lambda$ $\mathrm{DB}$, the versions that were current at the time we began the project three years ago, since we have made significant alterations and enhancements to these software.
The overall detailed implementation of the system is illustrated in Fig. 4. As illustrated, the schema declarations in ODL are first converted into SDL (the definition language provided on top of the SHORE storage manager), by $\lambda$-DB. The implementations of the schema declarations are stored in a separate source file that is compiled into a linkable library for the applications. Similarly, the query in the OQL format is typechecked, optimized and converted into an implementation of the optimal physical plan by $\lambda$-DB.

\subsection{Index key formats}

$\lambda$-DB generates the query implementation making use of its runtime interface to the SDL layer of SHORE. The query is evaluated in a streaming fashion, avoiding the materialization of the subqueries as much as possible. Indexing over object extents is achieved by maintaining a separate extent of indexes. In SHORE, the index objects have to reside within a "user level" object. Now, while $\lambda$-DB uses an ExtentIndex type to hold the indexes, it also converts all the index keys into a string format in order to handle them in a generic way. This turns out be a problem when handling keys that cannot be converted into character strings (such as in the case of spatial indexes),

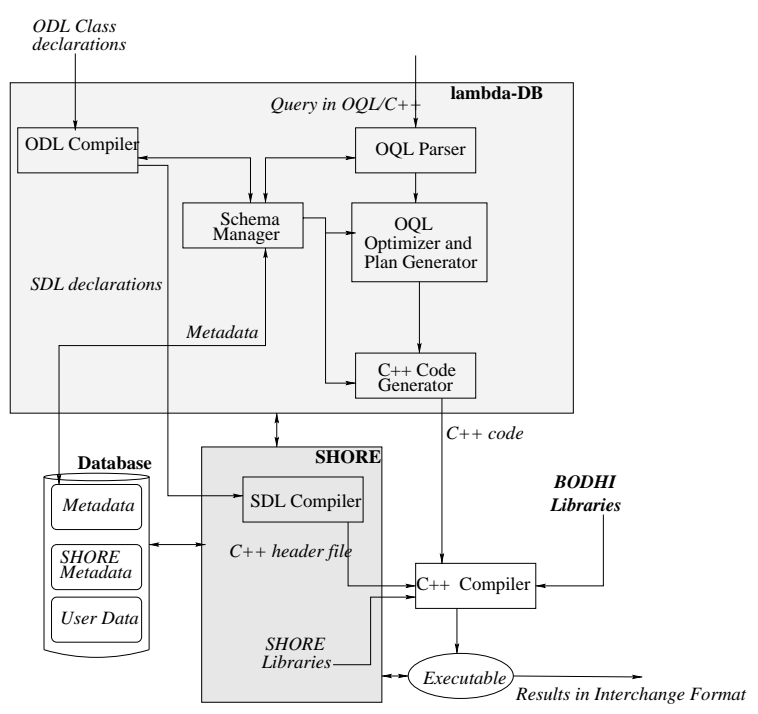

Fig. 4. Schema definition and query flow in BODHI. 
and in handling keys which result in a loss of information during the conversion (such as floating point values). Therefore, in order to support the spatial indexes from the ODL/OQL layers, we were forced to introduce a specialized key type for spatial indexes and also implement a special index holder class. This required a considerable amount of modification and extensions to the code in the query processor.

At the same time, the rule-based optimization scheme of the $\lambda$-DB simplified the process of adding new operators into OQL, as well as their optimization and rewritings into the physical operators based on the statistics. We added operators such as Overlaps, Inside, etc. for spatial operations, and sequence retrieval operators such as $B L A S T$ into the OQL specification supported by the query processor.

\subsection{Index visibility}

The implementation of access structures for spatial data and object hierarchies raised some of the subtle issues with regard to hosting them on the $\lambda$-DB and SHORE combination. One of the most surprising revelations was the lack of spatial index support at the SDL layer in SHORE - which is still not available since there have been no further releases of the SDL layer. The $R^{*}$-Tree is available only at the storage manager level, but is not exported to the SDL interface. This also meant that $\lambda$-DB which uses the SHORE through the SDL interface also has no knowledge of the spatial indexes. In order to provide the support at the OQL level we first had to rework the SHORE code, and then integrate it with the query processor.

\subsection{PD Index implementation}

While implementing the Path-Dictionary-based indexing for aggregation path queries, we found that the index structure as presented in [11] cannot be used in a stream based query processor such as $\lambda$-DB, without breaking the pipeline structure and materializing the query results at that join node. We addressed this problem by inverting the storage of paths to proceed from the top of the aggrega- tion tree instead of the suggested bottom-up approach. While this inversion may partially reduce the effectiveness of the Path-Dictionary, the major benefit of avoiding the huge cost of joins over object extents is retained.

We have extended the implementation given in [11] to support the additional requirements of allowing N:M relationships and presence of bags and sequences in the aggregation path. The main idea behind our extensions for the of $\mathrm{N}: \mathrm{M}$ relationships is to break them into multiple $1: \mathrm{N}$ relationships. But a straightforward application of this idea introduces complications in maintenance of s-expressions.

Supporting $N: M$ relationships: Consider the representative N:M relationship graph shown in Fig. 5(a). If we break this into multiple $1: \mathrm{N}$ relationships, the graphs and the corresponding s-expressions look as in Fig. 5(b). Note the redundancy in these s-expressions: The children of $B_{1}$ are replicated in both of the s-expressions of $A_{1}$ and $A_{2}$. This problem can be solved by using a flag in the entries of s-expression. The flag denotes whether the entry is a direct reference or an indirect reference. All the descendant entries of an OID will be stored only in the entry which contains direct reference to that OID. This modification is shown in form of a graph in

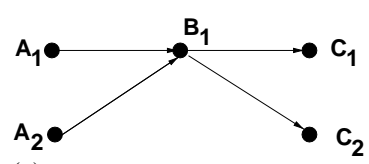

(a)
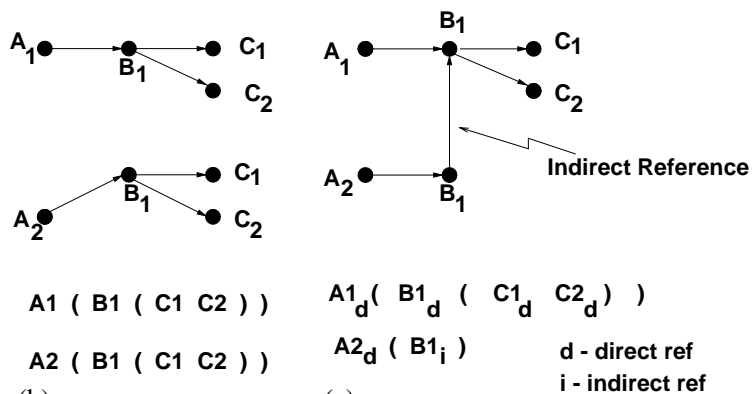

(b)

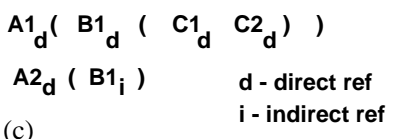

Fig. 5. Representing N:M relationships (a) N:M relationship (b) Equivalent $1: \mathrm{N}$ relationships with replicated paths (c) Equivalent 1:N relationships with indirect references. 
Fig. 5(c) with their corresponding s-expressions. Note that the suffix for each entry denotes whether it is a direct reference or an indirect reference. Though this modification duplicates (with different flag values) the $B_{1}$ entry, we avoid duplicating the children of $B_{1}$, thus saving space.

Extensions to support bags and sequences: The previous modification works fine for storing ordinary references and sets. But in the presence of bags, further redundancy is possible. The example for this is shown in Fig. 6. The number on the edge from $a$ to $b$ denotes the number of times $b$ appeared as a reference in the bag of $a$. The corresponding s-expressions for this graph using the above implementation are given in Fig. 6(b). Note that the entry of $B_{1}$ is repeated $n$ times in each expression, where $n$ denotes the number of times $B_{1}$ is referenced in the parent object. This replication can be eliminated by introducing one more field in the entry of s-expression which stores this replication count. This reduces the storage overhead for storing bags since OIDs are not duplicated. The s-expressions with this modification are shown in Fig. 6(c). The implementation also supports sequences by maintaining the order of the children of a given parent in the sexpressions.

\subsection{VAS feature}

In building the PD-index, we exploited the concept of Value Added Server (VAS), one of the strong features of SHORE. The ability to provide a concurrent storage manager with a full

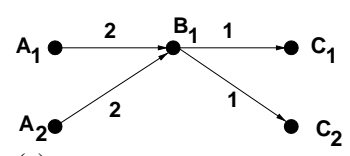

(a)

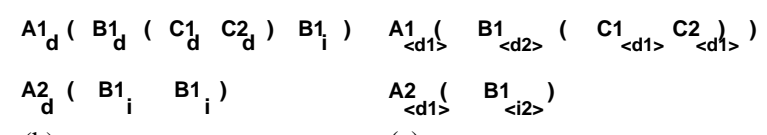

(b)

(c)

Fig. 6. Representing N:M relationships in presence of (a) Bags (b) Equivalent $1: \mathrm{N}$ relationships with indirect references (c) Equivalent $1: \mathrm{N}$ relationships with indirect references as well as replication counts. set of database features such as transactions, logging, recovery, etc., eased the task of extending the storage manager capabilities tremendously. Although RPC-based interaction between the storage server instances results in communication delays and reduced type-support across the storage servers, it enables cleaner separation of services provided by the storage manager.

We also used the VAS feature to provide genome sequence storage, and retrieval algorithms over this storage. An important advantage of this implementation is that it is easy to extend and optimize the sequence retrieval algorithms without affecting the rest of the system. A problem, however, was the following: The storage allocation of the sequences on the VAS is effected through a specific interface which stores the sequences in a compressed form on the disk. Ideally, this storage should be handled transparently. However, due to lack of post-construction hooks for object instantiation in $\lambda$-DB and SHORE, this compressed storage of sequences has to be explicitly called during database loading.

\section{Experimental results}

We have evaluated the performance of BODHI on a test-bed of typical queries in the bio-diversity domain. These queries make use of a mixture of synthetic and real datasets and consist of queries over both single-domain (such as taxonomy, spatial or sequence domains) and multiple domains-i.e., queries similar to Query 1 in the Introduction. Moreover, since spatial data forms a large fraction of data and is traditionally considered the main component of the query processing time, we studied the performance of the spatial component in detail. In particular, we evaluated the spatial data handling capabilities of BODHI over the datasets and queries of the Sequoia 2000 regional benchmark [17], a standard benchmark for spatial databases.

The performance numbers reported were generated on a Pentium-III $700 \mathrm{MHz}$ processor, with 512 MB memory and an 18 GB 10000 -RPM SCSI hard disk (IBM DDYS-T18350 M model), connected with Adaptec AIC-7896/7 Ultra2 SCSI host 
adapter. In order to reduce the effects of Linux's aggressive memory mapping of files, we flushed the benchmark database each time with an $\mathrm{I} / \mathrm{O}$ over a large database.

In the rest of the section, we first describe the synthetic datasets used in our queries, and then present BODHI's performance on these datasets for a variety of single-domain and multi-domain queries.

\subsection{Description of datasets}

The synthetic data used in our experiments conforms to a biodiversity object model, which is presented in part as an object diagram in Fig. 2. Even though we collaborated closely with the scientists of the ecological sciences in designing this object model to represent their requirements, we faced difficulties in procuring enough data to be used in the evaluation experiments of the system. This is because the domain experts have the bulk of their data in legacy formats, often on "herbarium sheets". 3 While we await the conversion of this data to electronic format, we have for the interim period created our datasets by boosting, with synthetic data, the limited real data that is currently available.

As shown in the object model, the schema is hierarchical in nature and consists of aggregation paths, inheritance structures over object types, spatial and genome sequence components. The well-known taxonomy aggregation path of OrderFamily-Genera-Species forms the backbone of the model. Each Species has a set of identifying characters (IdentChar), and there are many subcharacteristics that are inherited from this. The spatial component of the model consists of a collection of reported habitat areas for each Species. Also associated with each Species is a collection of DNA sequences that are used to study the evolutionary pathways amongst the species by locating homologies (sequences which have a high likelihood of sharing a common ancestor). We now describe the mechanism of

\footnotetext{
${ }^{3}$ These are sheets that contain a plant specimen and its details.
}

generating synthetic data that complies with the object model.

Taxonomy data: We generated the object relationships in taxonomy and characteristics hierarchy by setting a heuristic probability of association at each optional relationship. In case of collections in the aggregation path, the branch factor of the collection was uniformly distributed over specified end-points. The real data available for about 15 closely studied Plant species was boosted with this synthetic data.

Spatial data: We used the synthetic data generation method followed in [14]. The data consists of rectangular regions, whose centers are uniformly distributed over a unit square. The overlap between rectangular regions can be controlled by specifying the distribution of their height and width values. It should be noted that this dataset consists of only rectangular regions, while in reality we have to handle nonconvex polygonal regions as well. The performance of spatial data handling over real dataset (involving non-convex polygonal regions) will be evaluated separately through the Sequoia 2000 benchmark. Each species object generated above is associated with a synthetically generated polygon that represents the habitat of the species.

Genome data: In the case of Genome sequence data, we could use the data that is publicly available through repositories such as GenBank, SwissProt, etc. In our experiments, we made use of a randomly selected sample of "expressed sequence tags" (ESTs) of various genomes available from the BLAST database of EMBL GenBank [7]. We used these sequences to populate the DNA information of our synthetically generated species.

We summarize the parameters used for the benchmark dataset in Table 1 and the statistics of the resultant dataset in Table 2. We consider a set of 5 queries over this dataset that conforms to the schema illustrated in Fig. 2. These queries span the domains of taxonomy, spatial and genome data, and illustrate the capabilities of BODHI in handling these domains. In addition, the performance numbers of these queries provide an indicator towards overall expected performance of the system. 
Table 1

Parameters to synthetic data generator

\begin{tabular}{ll}
\hline Parameter & Value \\
\hline Branch factor at each level of taxonomy & $U(1,19)$ \\
$\begin{array}{l}\text { Mean (height, width) of habitat regions } \\
\text { Range of distribution of habitat regions }\end{array}$ & $\begin{array}{l}\text { From }(-100,-100) \\
\text { to }(-1000,-1000)\end{array}$ \\
No. of DNA sequences per species & 10 \\
\hline
\end{tabular}

Table 2

Statistics of the synthetic dataset

\begin{tabular}{lcc}
\hline Element & No. of tuples & Overall size (in KB) \\
\hline Order & 4 & 0.6 \\
Family & 46 & 7.1 \\
Genera & 496 & 76.0 \\
Species & 5155 & 1153.1 \\
FlowerChar & 5155 & 564.0 \\
Habitats & 5155 & 607.0 \\
InfloChar & 5 & 20.4 \\
EMBLEntry & 51550 & 2902 \\
& & 5330.2 \\
Total & &
\end{tabular}

\subsection{Biodiversity queries}

We now describe the set of queries considered to illustrate the capabilities of BODHI and present the performance numbers over each of these queries. The query mix can be split further into 3 categories: Taxonomy queries, Genome queries and Multi-Domain Queries. We collectively refer to Taxonomy and Genome Queries as Singledomain queries, since predicates involve either taxonomy hierarchy or genetic sequences associated with a species, but not both. The Multidomain queries, on the other hand, query across taxonomy hierarchy, habitat (spatial) collection and genetic sequences data corresponding to species. The performance numbers for the queries are summarized in Tables 3 and 4.

Taxonomy Query-1: Find all species that have the same Inflorescence characteristic in their Flowers as that of "Magnolia-champa". With reference to the bio-diversity data model shown in Fig. 2, this query performs a three level path traversal over the aggregation hierarchy of Species, Flower and
Table 3

Performance numbers for single-domain queries

\begin{tabular}{ll}
\hline Id & Time (min) \\
\hline Taxonomy Query-1 & 73 min (without path-dictionary) \\
& $0.5 \mathrm{~min}$ (with path-dictionary) \\
Genome Query-1 & $0.2 \mathrm{~s}$ \\
Genome Query-2 & $1.5 \mathrm{~min}$. \\
\hline
\end{tabular}

Table 4

Performance numbers for multi-domain queries

\begin{tabular}{lrcc}
\hline Id & $\begin{array}{l}\text { Without } \\
\text { index (s) }\end{array}$ & $\begin{array}{l}\text { Path-dictionary } \\
(\mathrm{s})\end{array}$ & $\begin{array}{l}\text { Spatial } \\
\text { path-dictionary (s) }\end{array}$ \\
\hline & & & \\
MDQ1 & 26.99 & 11.13 & 2.1 \\
MDQ2 & 553.66 & 542.12 & 530.2 \\
\hline
\end{tabular}

Inflorescence Characteristics. The performance results in Table 3 for this query show that without any indexing strategy for accessing the aggregation paths, the query execution times are unacceptably high - especially considering the modest size of the dataset. The performance of the query execution improves by two orders of magnitude with the presence of a Path-Dictionary index over the queried path. As discussed earlier in Section 4, the Path-Dictionary maintains a compact materialization of joins along the queried path, preventing the repeated computation of these expensive joins. Interestingly, if we follow the aggregation paths through the usage of "swizzled pointers" available through $\mathrm{C}++$ interface of SHORE, this query can be answered in $8.5 \mathrm{~s}$, which is much faster than even using Path-Dictionary based indexing. It has to be noted that rewritings available in query-processors such as $\lambda$-DB do not make use of these features available with the storage managers, thus incurring heavy cost of joins.

Genome Query-1: Retrieve all DNA sequences of Magnolia-champa. The DNA sequences are stored encoded, using context-free encoding, in a separate storage. This encoding increases the disk-memory bandwidth and enables the sequence similarity algorithms to operate in this encoded domain 
itself. At the same time, there is an overhead of decoding them before presenting to the user. The performance numbers of this query give estimate of the delay involved in decoding these sequences.

Genome Query-2: List names of all Species that have a DNA sequence within a BLAST score of 70 with any sequence of Magnolia-champa. The computation of BLAST scores over a database could be a time consuming task. We don't have any indexing schemes for speeding these queries, for reasons mentioned earlier in Section 3, and hence for each query sequence we have to make a full scan of the sequence database and compute the scores, significance of the alignments, etc. The timing for this query - which results in 10 BLAST computations - is about $1.5 \mathrm{~min}$, as mentioned in Table 3. When this number is compared against the query capabilities of BLAST-farms run by organizations such as EMBL, it might look rather high. However, BLAST-computation farms make use of large-scale and heavily optimized data handling equipment and keep the entire database in memory for speeding up the processing times, while BODHI is aimed to handle varied data, and is running on a general purpose small-scale machine.

Multi-domain Query-1: Find all Species sharing a common habitat and having the same Inflorescence characteristic as Magnolia-Champa. This query, which is common among ecologists, is targeted at the combination of hierarchical data of Taxonomy domain, and associated Spatial data. The query evaluates the combined effectiveness of the PathDictionary index and $R^{*}$-Tree indexes available in BODHI. The performance numbers provided in Table 4 are for the optimal query plan which performs the spatial overlap before computing the joins over the aggregation paths. Since spatial overlap is highly selective in the existing dataset, the number of path aggregation traversals are reduced to a very small number. As a result, we see that even though this query is more complicated than Taxonomy Query-1, it takes less than $0.6 \%$ of time taken for Taxonomy Query-1 even in the absence of the Path-Dictionary index. The presence of Path-Dictionary reduces the execution time further, from $26.99 \mathrm{~s}$ to $11.13 \mathrm{~s}$ - a reduction of $58 \%$. In this case, the execution times are dominated by the spatial overlap computation. We can see this clearly by looking at the performance of the query when both $R^{*}$-Tree and PathDictionary are present. The query time is just around $2 \mathrm{~s}$, almost an $80 \%$ improvement. This clearly indicates that both indexing strategies are extremely useful for such queries.

Multi-domain Query-2: Retrieve all pairs of Species sharing a common habitat, having same Inflorescence characteristic and having a DNA sequence within BLAST score of 70 of each other. This query, which extends the Multi-domain Query-1 by adding an extra predicate for the BLAST score computation for each of the sequences in the target species, is similar to the "goal" query that we presented earlier as Query 1 in the Introduction. The OQL version of this query, which is what is input to the BODHI system, is given below:

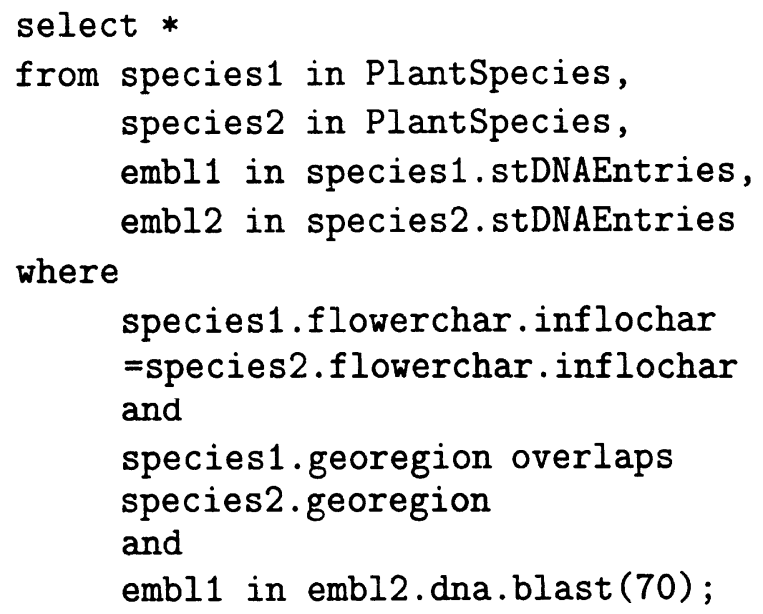

Referring to Table 4, we see that the execution times are much higher than those of Multi-domain Query-1-due to the additional 50 BLAST computations. The reduction in execution times are approximately same as in Multi-domain Query-1, about $11 \mathrm{~s}$ in presence of Path-Dictionary and by a further $10 \mathrm{~s}$ in presence of both $R^{*}$-Tree and PathDictionary indices. Hence, this query is clearly dominated by the BLAST computations. Therefore, it appears that it is imperative to develop indexing strategies to improve performance of such queries over genome sequence data. 


\subsection{Evaluating spatial data handling}

The evaluation of queries over spatial data has traditionally been considered as a highly computeintensive operation, and many indexing strategies have been proposed to improve the performance of these queries. The SEQUOIA benchmark has been quite popular for evaluating the performance and capabilities of spatial databases. It consists of a set of 10 queries over a schema involving the spatial objects (such as polygons, points and graphs) and also bitmap (raster) objects. As we do not have support for bitmap data formats in BODHI, we have chosen to ignore the raster dataset and queries (2), (3), (4) \& (9), which involve these objects. The vector benchmark data consists of 62556 Point objects, 58585 Polygons and 201659 Graph objects. Table 5 summarizes the response times (in seconds) for the queries on this data. We have compared BODHI's performance with Paradise [18], a spatial database system also built on the SHORE storage manager, and Postgres [35], a popular free object-relational database. The numbers given for these two systems are taken from those reported in [18].

The SEQUOIA benchmark results in Table 5 show that BODHI is very close in performance to that of Paradise, which is a specialized and highly optimized spatial database system. Even though the hardware platform used by the two systems are difficult to compare, it should be noted that both Paradise and BODHI use the same underlying storage manager. In addition the following points regarding numbers reported under BODHI should be noted: (i) We use file-based storage management instead of using raw-disk as done by Paradise system; (ii) The optimal physical query plan is generated through a generic object-oriented query processor; (iii) The type-system is user-defined whereas in Paradise the basic type system of SHORE has been augmented; and, (iv) the size of the buffer pool used by SHORE is the default value-320 KB, whereas Paradise used $16 \mathrm{MB}$.

We now present the chosen set of SEQUOIA queries and their performance statistics. We also explain a few of these queries and highlight their importance in a typical set of bio-diversity query workloads. For detailed explanation and analysis of all the queries we refer the reader to [36].

Sequoia 1-dataloading and index creation: This query populates the database from a given set of datafiles, and is expected to exercise the bulkloading facility in the database. At the time of writing, we still do not have the bulk-loading feature in BODHI, resulting in a transaction commit for each object hierarchy. Therefore, the table represents only an upper bound on the dataload and indexing times for the spatial component. Referring to Table 5, we see that this is the only benchmark query in which BODHI is far worse than Paradise which supports bulkloading facility. However, we do not see it as a major bottleneck in BODHI, since the biodiversity databases are not expected to have high rates of bulk data updates. Instead, these databases are highly query-intensive and hence it is important to have fast query processing speeds. In addition, we expect improvements in performance when the bulk-loading scheme is put in place for BODHI.

Sequoia 5-Select a point based on its name.

Table 5

SEQUOIA Benchmark numbers (in seconds)

\begin{tabular}{rllcr}
\hline Id & $\begin{array}{l}\text { BODHI } \\
\text { (with R*-Tree) }\end{array}$ & $\begin{array}{l}\text { BODHI } \\
\text { (with Hil. R-Tree) }\end{array}$ & Paradise & Postgres \\
\hline 1 & 5742.0 & 4662.0 & 3613.0 & \\
\\
5 & $\left(\mathrm{R}^{*}\right.$-Tree: 1342.0$)$ & $($ Hil. R-Tree: 262.0$)$ & 0.2 & 0.9 \\
6 & 0.12 & 0.11 & 7.0 & 36.0 \\
7 & 8.0 & 8.0 & 0.6 & 30.5 \\
8 & 0.66 & 0.7 & 9.4 & 62.2 \\
10 & 9.7 & 9.6 & Not supported & 327.2 \\
\hline
\end{tabular}


Sequoia 6-Select polygons overlapping a specified rectangle: This is one of the typical spatial queries asked in ecological studies where a geographic region is split into a set of grids and the researchers would want to identify the species whose previously recorded habitat boundaries overlap with the grid being studied. This could be important in identifying species whose coexistence in a region is to be targeted for study. The performance of spatial operators such as overlap depend directly on the performance of implementing these operators on a spatial index such as $R^{*}$-Tree or Hilbert R-Tree. Since the $R^{*}$ Tree implementation of BODHI is the same as that of Paradise (both use the index provided by the SHORE storage manager), we do not see much difference in the query execution performance.

Sequoia 7-Select polygons greater than specified area, contained within a circle: We see similar queries occurring in bio-diversity studies with variations in the area selection clause of the query. The area of a polygon is provided through a derived attribute - computed based on the coordinates of the polygon. This is extendible to allow for selection over arbitrary derived attributes over which an index can be built. Thus, in ecological study databases, we get variations of the query that locate all the habitats that are near a study center, with a derived attribute value (such as bio-mass index of the habitat, etc.).

This query reflects the combination of B-Tree and spatial index based query processing. The order in which this query gets evaluated - whether the B-Tree lookup or the $R^{*}$-Tree based overlap selection is made as the first step - makes a big difference in the query answering times. The usage of query optimizer which maintains cost statistics and uses it to arrive at the final evaluation order is also tested in this query. The numbers presented in Table 5 are for the optimal plan generated by the query processor of BODHI, which is to perform the $R^{*}$-Tree based overlap selection first and then the B-Tree-based polygon area selection.

Sequoia 8-Select polygons overlapping a rectangular region around a point.

Sequoia 10-Select points contained in polygons with specific landuse type.
We also executed the above Sequoia benchmark queries with Hilbert R-Tree in place of $R^{*}$-Tree. The results obtained are shown in Table 5. The building times of Hilbert R-Tree were quite low in comparison to that of $R^{*}$-Tree, and at the same time provide almost the same performance. The numbers shown are for Hilbert R-Tree which employs s-to-(s +1$)$ split policy on overflow, with $s=2$. Even though the performance of the Hilbert $\mathrm{R}$-Tree could be improved by increasing the value of $s$, the index creation times increase sharply with $s$. Hence, the current choice of split policy was chosen to optimize on the index building time and the performance of the index over benchmark queries.

\section{Related work}

Bio-diversity data consists of both macro-level and micro-level information ranging from ecological information to genetic makeup of organisms and plants. Apart from our work, we are not aware of any other that attempts to combine the complete spectrum of information, though the need for it is highlighted in a recent proposal for Global Bio-diversity Information Facility $(G B I F)$ [22] by Organization for Economic Co-operation and Development (OECD). This proposal identifies the domain level challenges in building a global, interconnected data repository of biodiversity information systems and notes that the urgent requirement in bio-diversity studies is a suitable information management architecture for handling vast amounts of diverse data.

In the area of macro-level bio-diversity data management, there have been many governmental efforts from various countries such as ERIN [37], INBio [38] and some global initiatives such as Species 2000 [39], the Tree of Life [40], etc. And in a recent report sponsored by the National Science Foundation in the USA [41], a group of computer scientists have outlined research directions in bioinformatics.

The micro-level bio-diversity data, or genetic information of various species, has been growing steadily due to the multitude of genome sequencing initiatives. The specific data management 
issues in handling such data $[42,43]$ have been addressed in quite a few proposals. In all of these proposals, the database management architecture has been tailored for the specific purposes of the project. Consider the $A C e D B$ (A C.elegans Database) [44] database system, originally proposed for the $C$. elegans genome sequencing project. ACeDB is an object oriented data management tool that has many features, including the handling of missing data and schema evolution issues, that make it an extremely popular software in many sequencing projects. However, in spite of its popularity in the genome sequencing community, it cannot be considered for the larger requirements of bio-diversity data handling due to the following reasons: (1) Its lack of support for geo-spatial data; (2) Weak support for database updates; and (3) The lack of recovery mechanisms necessary in large data repositories.

In BODHI, we have provided the key strengths of ACeDB (its sequencing algorithms and objectoriented basis), and augmented it with the strong database functionalities and the related features that are necessary for a complete bio-diversity information repository.

\section{Conclusions}

We have reported in this paper on our experiences in building BODHI, an objectoriented database system intended for use in bio-diversity applications. To the best of our knowledge, BODHI is the first system to provide an integrated view from the molecular to the organism-level information, including taxonomic data, spatial layouts and genomic sequences.

BODHI is operational, completely free and is built around publicly available software components and commodity hardware. Further, BODHI incorporates a variety of indexing strategies taken from the recent research literature for efficient access of different data types. Through a detailed performance study using a range of biological queries, we showed that these indexes were extremely effective in reducing the running times of the queries. Our experiments also showed that while spatial operations are certainly expensive as mentioned in the literature, it is perhaps the genomic sequencing queries that are really the "hard nuts" in the biological context. Therefore, the importance of developing efficient indexing strategies for sequence data cannot be overemphasized.

We hope that BODHI can be successfully used by biologists as the central information repository of their workbench, and by computer scientists as a realistic test-bed for evaluating the efficacy of their algorithms. We are currently working on adding new indexing mechanisms such as the Pyramid Technique [45] for indexing high-dimensional data, where each data object has thousands of attributes - such data is especially common in drug-related datasets.

\section{Appendix A. Typical tagged output from BODHI}

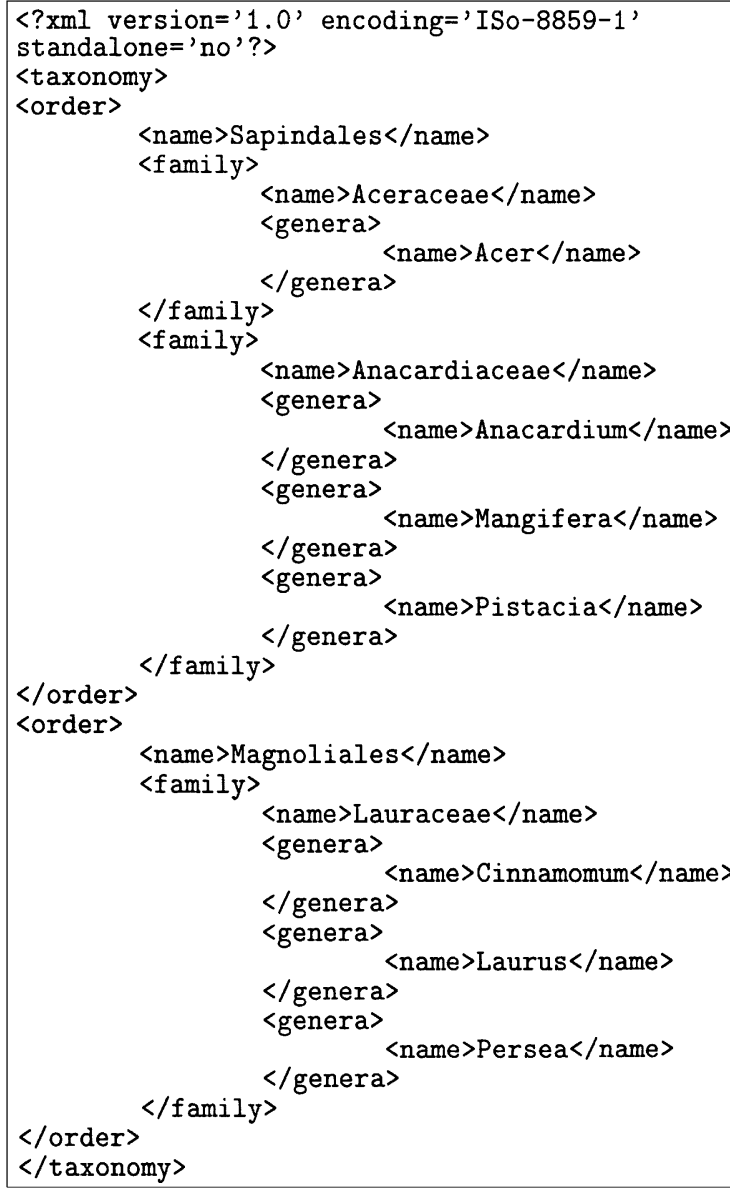




\section{Appendix B. Graphical query form of BODHI}

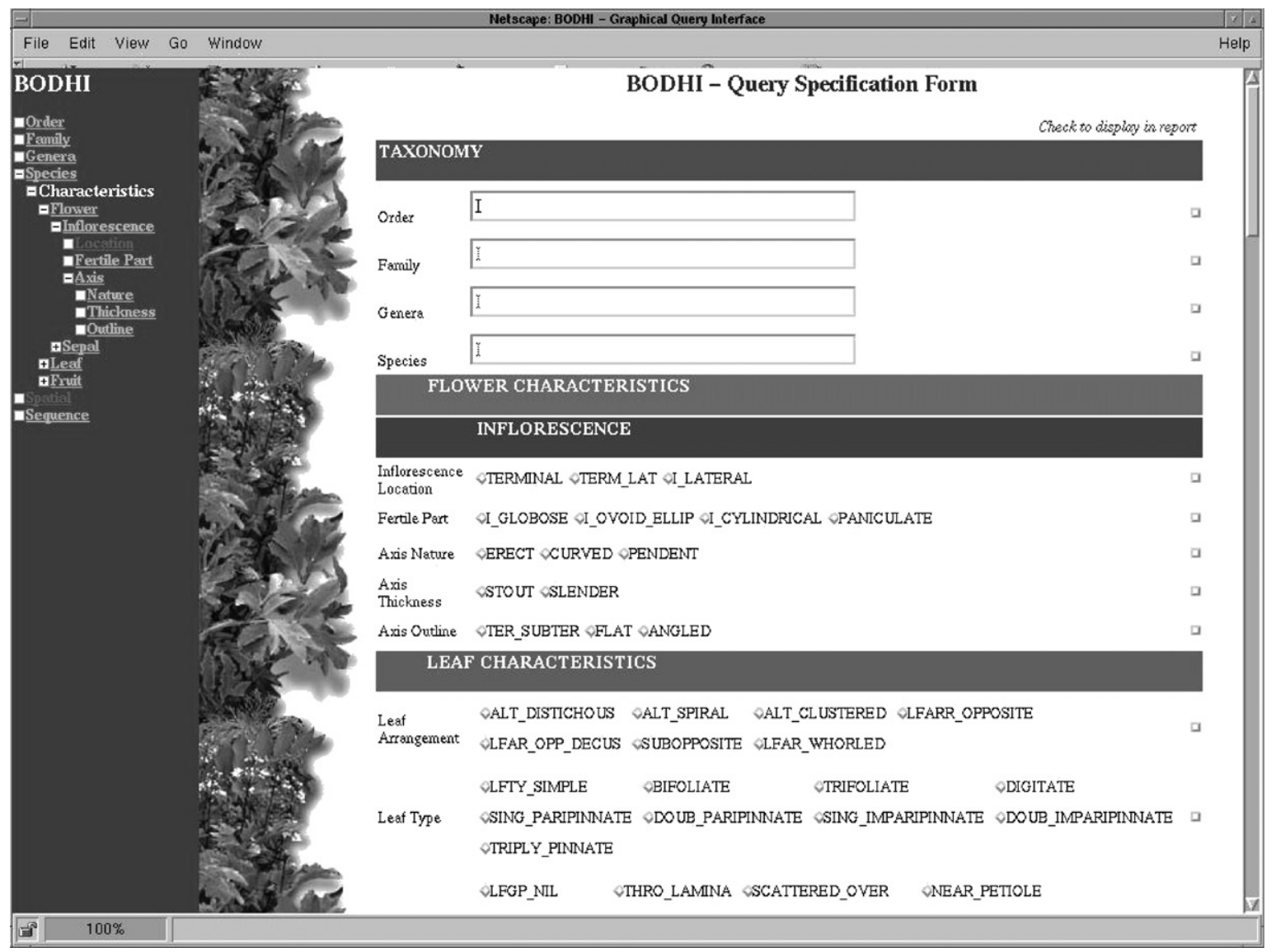

\section{Acknowledgements}

This work was supported by a research grant from the Department of Bio-technology, Govt. of India. Profs. V. Nanjundiah and Madhav Gadgil of the Centre for Ecological Sciences, Indian Institute of Science, have been co-investigators on the project. Several ME students from the Department of Computer Science \& Automation, Indian Institute of Science, have participated at various stages in the BODHI project implementation.

\section{References}

[1] M.A. Lane, J.L. Edwards, E. Nielsen, Biodiversity informatics: the challenge of rapid development, large databases, and complex data (keynote), in: VLDB 2000, Proceedings of the 26th International Conference on Very Large Data Bases, 2000.

[2] World Conservation Monitoring Center, http://www. wcmc.org.uk/.

[3] T. Mitchellolds, Does environmental variation maintain genetic variation - a question of scale, Trends Ecology Evolution 7 (1992) 397-398.

[4] J. Viescas, Running Microsoft Access 2000, Microsoft Press, 1999.

[5] Getting to Know arcview gis for version 3.1, 1999.

[6] S. Altschul, W. Gish, W. Miller, E.W. Myers, D. Lipman, A basic local alignment search tool, J. Mol. Biol. 215 (1990).

[7] GenBank, ftp://www.ncbi.nlm.nih.gov/.

[8] J.C. Setubal, J. Meidanis, Introduction to Computational Molecular Biology, PWS Publishing Company, 1997.

[9] M.J. Carey, D.J. DeWitt, M.J. Franklin, N.E. Hall, M.L. McAuliffe, J.F. Naughton, D.T. Schuh, M.H. Solomon, C.K. Tan, O.G. Tsatalos, S.J. White, M.J. Zwilling, 
Shoring up persistent applications, in: Proceedings of the 1994 ACM SIGMOD International Conference on Management of Data, 1994.

[10] L. Fegaras, An experimental optimizer for OQL, Technical Report TR-CSE-97-007, University of Texas at Arlington, 1997.

[11] W. Lee, D.L. Lee, Path dictionary: a new access method for query processing in object-oriented databases, IEEE Trans. Knowledge Data Eng. 10 (3) (1998).

[12] T.A. Mück, M.L. Polaschek, A configurable type hierarchy index for OODB, VLDB J. 6 (4) (1997).

[13] N. Beckmann, H.-P. Kriegel, R. Schneider, B. Seeger, The $R^{*}$-tree: an efficient and robust access method for points and rectangles, Proceedings of the 1990 ACM SIGMOD International Conference on Management of Data, 1990.

[14] I. Kamel, C. Faloutsos, Hilbert R-tree: an improved R-tree using fractals, in: VLDB'94, Proceedings of the 20th International Conference on Very Large Databases, 1994.

[15] R.G.G. Cattel (Ed.), The Object Database Standard: ODMG-93, Morgan Kaufmann Publishers, Los Altos, CA, 1994.

[16] S.J. Bedathur, J.R. Haritsa, Design and implementation of a biodiversity information management system, in: Proceedings of the Tenth International Conference on Management of Data, 2000.

[17] M. Stonebraker, J. Frew, K. Gardels, J. Meredith, The SEQUOIA 2000 storage benchmark, in: Proceedings of the 1993 ACM SIGMOD International Conference on Management of Data, 1993.

[18] D.J. DeWitt, N. Kabra, J. Luo, J.M. Patel, J.B. Yu, Clientserver paradise, in: VLDB'94, Proceedings of the 20th International Conference on Very Large Data Bases, 1994.

[19] R.J. Pankhurst, Practical Taxonomic Computing, Cambridge University Press, Cambridge, 1991.

[20] D.J. Lipman, W.R. Pearson, Rapid and sensitive protein similarity searches, Science 227 (1985).

[21] Scaleable similarity searching, http://www.research.ibm.com/compsci/compbio/scaleable.html.

[22] H. Saarenmaa, The global biodiversity information facility: architectural and implementation issues, Technical Report TR-34, European Environment Agency, 1999.

[23] ANZMETA DTD Version1.1, http://www.erin.gov.au/ database/metadata/anzmeta/anzmeta-1.1.html.

[24] Biopolymer Markup Language-BIOML, http://www. bioml.com.

[25] L. Wang, P. Rodriguez-Tomé, N. Redaschi, P. McNeil, A. Robinson, P. Lijnzaad, Accessing and distributing EMBL data using CORBA, http://genomebiology.com/2000/1/5/ research/0010, 2000.

[26] R.H. Güting, An introduction to spatial database systems, VLDB J. 3 (4) (1994).

[27] A. Kemper, C. Kilger, G. Moerkotte, Function materialization in object bases, in: Proceedings of the 1991 ACM SIGMOD International Conference on Management of Data, 1991.
[28] G. Graefe, R.L. Cole, D.L. Davison, W.J. McKenna, R.H. Wolniecwicz, Extensible query optimization and parallel execution in volcano, in: J.C. Freytag, D. Maier, G. Vossen (Eds.), Query Processing for Advanced Database Applications, Morgan Kaufmann, Los Altos, CA, 1993.

[29] C. Mohan, D.J. Haderle, B.G. Lindsay, H. Pirahesh, P. Schwarz, ARIES: a transaction recovery method supporting fine-granularity locking and partial rollbacks using write-ahead logging, TODS 21 (4) (1992).

[30] P. Seshadri, M. Paskin, PREDATOR: An OR-DBMS with enhanced data types, in: Proceedings of the 1997 ACM SIGMOD International Conference on Management of Data, 1997.

[31] Dbworld mailing list, http://www.cs.wisc.edu/dbworld.

[32] T. Kahveci, A.K. Singh, An efficient index structure for string databases, in: VLDB 2001, Proceedings of the 27th International Conference on Very Large Data Bases, 2001.

[33] E. Hunt, M.P. Atkinson, R.W. Irving, A database index to large biological sequences, in: VLDB 2001, Proceedings of the 27th International Conference on Very Large Data Bases, 2001.

[34] Extensible markup language, http://www.w3.org/TR/ REC-xml.

[35] M. Stonebraker, L.A. Rowe, The design of postgres, in: Proceedings of the 1986 ACM SIGMOD International Conference on Management of Data, 1986.

[36] S.J. Bedathur, J.R. Haritsa, U. Sankar Sen, The building of BODHI, a bio-diversity database system, Technical Report TR-2001-02, DSL, Indian Institute of Science, 2001.

[37] T. Boston, D. Stockwell, Interactive species distribution reporting, mapping and modeling using the World Wide Web, in: Proceedings of the Second International WWW Conference, 1994.

[38] INBioparque, http://www.inbio.ac.cr/en/default.html.

[39] Species2000, http://www.species2000.org/.

[40] D.R. Maddison, W.P. Maddison, The tree of life: a multiauthored, distributed Internet project containing information about phylogeny and biodiversity, http://phylogeny.arizona.edu/tree/phylogeny.html, 1998.

[41] Research Directions in Biodiversity and Ecosystem Informatics, Report of an NSF, USGS, NASA Workshop on Biodiversity and Ecosystem Informatics, 2001.

[42] N. Goodman, S. Rozen, L. Stein, Building a laboratory information system around a $\mathrm{C}++$-based object oriented DBMS, in: VLDB'94, Proceedings of the 20th International Conference on Very Large Data Bases, 1994.

[43] N. Goodman, S. Rozen, L. Stein, A glimpse at the DBMS challenges posed by the human genome project, ftp: //genome.wi.mit.edu/pub/papers/Y1994/challenges.ps.Z.

[44] R. Durbin, J. Thierry-Mieg, A C.elegans Database Documentation, ftp://ncbi.nlm.nih.gov.

[45] S. Berchtold, C. Böhm, H.-P. Kriege, The pyramidtechnique: towards breaking the curse of dimensionality, in: Proceedings of the 1998 ACM SIGMOD International Conference on Management of Data, 1998. 